\title{
$\mathfrak{I} \mathfrak{j} \mathfrak{a} \mathfrak{l}$.
}

\section{$\subseteq \mathfrak{i} \mathfrak{u} \mathfrak{g} \mathfrak{c} \mathfrak{d} \mathfrak{i} \mathfrak{d} \mathfrak{t} \mathfrak{e}$.}

Sclle

1. Die Sinngebidite an ben lejer. . . . . . . . . . . . . . . 5 5 Ebendicjciben. . . . . . . . . . . . . . . . . . . 5

3. Tuf ben neucrn zigelt blejer Einngebiale . . . . . . . . . . . . 5

4. Der Etadelteint. . . . . . . . . . . . . . . . . . . 6

5. Nilanber . . . . . . . . . . . . . . . . . . 6

6. In ben Marull . . . . . . . . . . . . . . . . . . . . . . 6

7. Merhar und 2 mor . . . . . . . . . . . . . . . . . . . . 6

8. Thtar unb Etar . . . . . . . . . . . . . . . . . . . . . . T

0. Der geizige Didjter. . . . . . . . . . . . . . . . . T

10. Puf Qucinten . . . . . . . . . . . . . . . . . . . . . 7

11. Iuf bie Futopa. . . . . . . . . . . . . . . . . . . . . 7

12. Pompils Lambgitt . . . . . . . . . . . . . . . . . 8

13. Miberruf bes Boriget . . . . . . . . . . . . . . . s

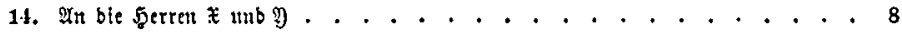

15. Dic Froibleit getwifjer Gebidte. . . . . . . . . . . . . . 9

16. Itnf bas Jungiernftift zu**. . . . . . . . . . . . . . . . 9

17. ?n ber Doltor Sp** . . . . . . . . . . . . . . . . . . . 9

18. Iuf ben Muemon . . . . . . . . . . . . . . . . . 9

19. Bavs Gajt. . . . . . . . . . . . . . . . . . 10

20. ILf ben $\mathfrak{n u f u s . ~ . ~ . ~ . ~ . ~ . ~ . ~ . ~ . ~ . ~ . ~ . ~ . ~ . ~ . ~ . ~ . ~ . ~} 10$

21. $\mathfrak{A n f}$ Dorinben . . . . . . . . . . . . . . . . . . 10

22. शT bas Bitb ber Beredtigleit in bem 5aule etres

23. 2uf eitret abeltgen Dummloyf . . . . . . . . . . . . . . . 11 
Geile

24. Â eine wïtbige Pribatperion . . . . . . . . . . . . . . . . 11

25. Auf bie gris . . . . . . . . . . . . . . . . . . . . . 11

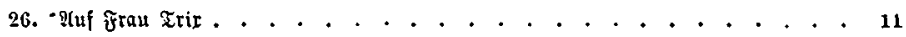

27. Suf futrizs corab . . . . . . . . . . . . . . . . . . 12

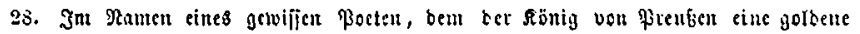
Dofe ldjente . . . . . . . . . . . . . . . . . . . . 12

29. Tuf beu faljden Muf bou Nigrius zobe . . . . . . . . . . . . . 12

30. Saf ben sargil . . . . . . . . . . . . . . . . . . 12

31. Dil $\tilde{\delta}^{\top n d j t}$. . . . . . . . . . . . . . . . . . . . 13

32. Dic \$oblthatent . . . . . . . . . . . . . . . . . 13

33. In eiren Betigigen . . . . . . . . . . . . . . . . . . . . 13

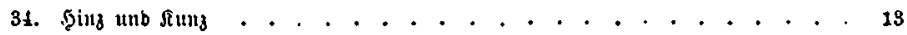

35. 2tuf eine tange গlaje . . . . . . . . . . . . . . . . . 14

36. Ituf હtipfen . . . . . . . . . . . . . . . . . . . . 14

37. Tuf ben Gantulus . . . . . . . . . . . . . . . . . . . 14

38. Th Griflen . . . . . . . . . . . . . . . . . . . 15

39. In ben Galomon . . . . . . . . . . . . . . . . 15

40. Tuf ebendenfelben . . . . . . . . . . . . . . . . . 15

11. Das böle getí . . . . . . . . . . . . . . . . . 15

42. Nn ben Pemil . . . . . . . . . . . . . . . . . . 16

43. Trur an ben Gabil . . . . . . . . . . . . . . . . . . 16

4t. Fntwort bes Sabin . . . . . . . . . . . . . . . . . 16

45. $\mathfrak{2}$ ethen Qügnet . . . . . . . . . . . . . . . . . . . . 16

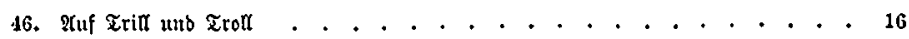

47. Entjacibung res Morigen . . . . . . . . . . . . . . . . 17

43. In bie *. . . . . . . . . . . . . . . . . . 17

49. IUf $\mathfrak{A}$ futberı . . . . . . . . . . . . . . . . . . 17

50. Auf eirtet 21 ranb $\jmath^{*} *$. . . . . . . . . . . . . . . . 17

51. Itn Einen . . . . . . . . . . . . . . . . . . . . . 18

52. Grabjhtift bes ßitulus . . . . . . . . . . . . . . . . 18

53. Muf belt Sobgll . . . . . . . . . . . . . . . . . . 18

54. Atu ben \$ompil . . . . . . . . . . . . . . . . . 18

55. Aluf ben tob eitte $\mathfrak{i f f e n ~ . ~ . ~ . ~ . ~ . ~ . ~ . ~ . ~ . ~ . ~ . ~ . ~ . ~ . ~ . ~} 18$

56. Grabjdrift auf eberbenfelbent . . . . . . . . . . . . . . 19

57. Suf bie $\$$ gafiz . . . . . . . . . . . . . . . . . . . . 19

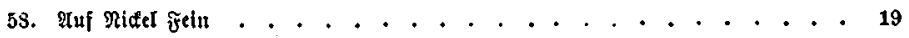

59. Tuf eine Siebgaberin bes retaueripiels . . . . . . . . . . . . . . 19

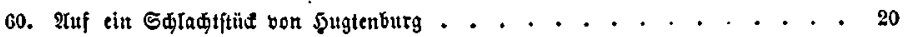

61. Nuf ben 5abtabor . . . . . . . . . . . . . . . . . 20

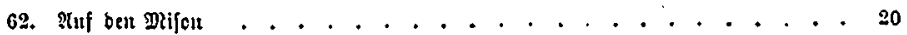


Eeitc

63. Der reidje freier. . . . . . . . . . . . . . . . . . . . . 20

64. Auf ben Rufinus . . . . . . . . . . . . . . . . . . . . . . 20

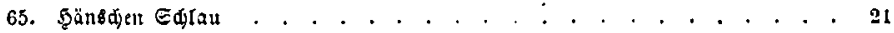

66. In ble oorifts . . . . . . . . . . . . . . . . . . . 21

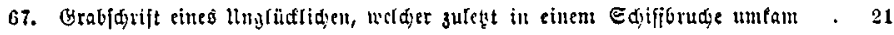

68. In einen folediten mater. . . . . . . . . . . . . . . . . . . 21

69. P(uf cine Bitbfäule ocs ?tmor . . . . . . . . . . . . . . . . . 22

70. 2ni tbenblefelbe . . . . . . . . . . . . . . . . . . . . 22

71. Iuf cbenbiefelbc . . . . . . . . . . . . . . . . . . . . $\mathbf{2}$

72. शhf cbenbieiclbe . . . . . . . . . . . . . . . . . . . . . . 22

73. 2uf ebenbisfelle . . . . . . . . . . . . . . . . . . . . 22

7. Ituf ben fobull . . . . . . . . . . . . . . . . . . . . . . 23

75. Iง ben trägen ! . . . . . . . . . . . . . . . . . . . . . . $\mathbf{2 3}$

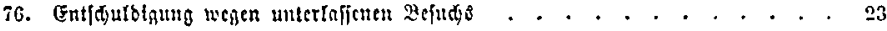

7. In ben paul . . . . . . . . . . . . . . . . . . . . . . . $\mathbf{2 t}$

73. Selt unb Polt . . . . . . . . . . . . . . . . . . . . . . 24

79. Der trante Etar. . . . . . . . . . . . . . . . . . . . . . 24

80. Die brante Salto. . . . . . . . . . . . . . . . . . . 24

81. Der ธđufter franz . . . . . . . . . . . . . . . . . . 25

32. Das Măbdell. . . . . . . . . . . . . . . . . . 25

83. Iuf ber fiell . . . . . . . . . . . . . . . . . . . . . . . 26

84. In ben நerru $D^{*}$. . . . . . . . . . . . . . . . . . $\mathbf{2 6}$

85. ITn eilien getaigen Mater . . . . . . . . . . . . . . . . . . . 26

86. STuf ben Saltz . . . . . . . . . . . . . . . . . . . . . 26

87. Tuf ben Supan. . . . . . . . . . . . . . . . . . . 26

88. An ben Sefer . . . . . . . . . . . . . . . . . . . . 27

89. In ben setrn von Dampi . . . . . . . . . . . . . . . . . . 27

90. Un cbenbenjelben . . . . . . . . . . . . . . . . . . . . 27

91. Tuf einen gersifjen Didter . . . . . . . . . . . . . . . . . פ

92. Itn ben $\mathfrak{B e}$. . . . . . . . . . . . . . . . . . . . . . . 28

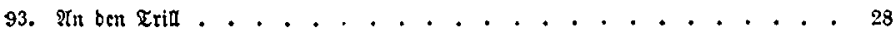

91. Itr ebenbenjetsert. . . . . . . . . . . . . . . . . . 23

95. 2 A bie $\mathfrak{F} u$ thta . . . . . . . . . . . . . . . . . . . . 23

96. Inf ben xat bes D. Dleab . . . . . . . . . . . . . . . 23

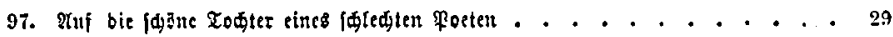

93. Iuf ericndiejetle. . . . . . . . . . . . . . . . . . . 29

99. Ituf ben Gestus . . . . . . . . . . . . . . . 29

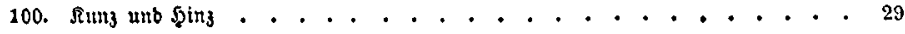

101. Tuf ben \$av . . . . . . . . . . . . . . . . 30

102. Inl Dorinber . . . . . . . . . . . . . . . 30 
Srite

103. Ituf bie chatatgec . . . . . . . . . . . . . . . . . \$0

104. Yuf btc 5 ülte kes Jrus . . . . . . . . . . . . . . . . . . 30

105. Iuf einen geriijent geidatutebuer . . . . . . . . . . . . . . . . 30

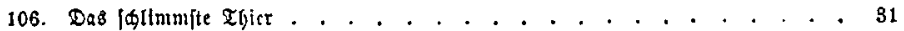

107. Ituf bie Magbalis . . . . . . . . . . . . . . . . . . . 31

103. Tuf gordet . . . . . . . . . . . . . . . . . . 31

109. Fftmps . . . . . . . . . . . . . . . . . . . 31

110. Der Ipieliüditine Deutidge . . . . . . . . . . . . . . . . . . 31

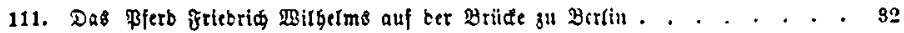

112. 2tuf ble felge Mumma . . . . . . . . . . . . . . . . . . . 32

113. Fine Bejunbgeit anf ble Gejunblyciter . . . . . . . . . . . . . 32

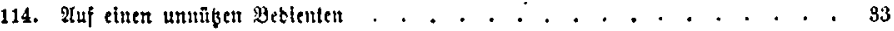

115. Der Efinur . . . . . . . . . . . . . . . . . . . . . . . 38

116. Themis über ifr Bitenis in ben 5aule eittes Midtero . . . . . . . . 33

117. Det frutdtfaute . . . . . . . . . . . . . . . . . . . . 33

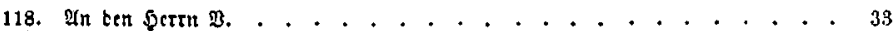

119. Puf bie Genefung elter Bubleria . . . . . . . . . . . . . . St

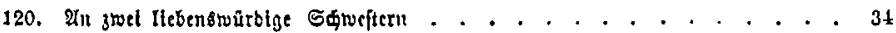

121. थn ben Stlius . . . . . . . . . . . . . . . . . . . . . . 3t

122. Uuf ben D. sityitill . . . . . . . . . . . . . . . . . . 34

123. Iuf Mulfetı . . . . . . . . . . . . . . . . . . . . . . . 85

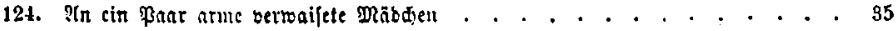

125. 2n bell Bar . . . . . . . . . . . . . . . . . . 35

126. Nuf ben Cytgatift . . , . . . . . . . . . . . . . . . . . . 35

127. Der befte Wurf. 2t1 cin Paar Brettipleter . . . . . . . . . . . . 96

128. Uuf ben Maler filcts . . . . . . . . . . . . . . . . . 36

129. Tuf circu 3meitumpf . . . . . . . . . . . . . . . . . . . 36

180. 2uf ben $\mathfrak{l}$ rin . . . . . . . . . . . . . . . . . . . . . 36

131. Stuf ben Btit . . . . . . . . . . . . . . . . . . . 36

132. Die DorfpleIe ber Berjögnutig . . . . . . . . . . . . . . . . 37

133. Auf ben \$frlem . . . . . . . . . . . . . . . . . . 37

134. Suf ber গTwar . . . . . . . . . . . . . . . . . . . 37

135. Seufaer cines firanten . . . . . . . . . . . . . . . . . 37

186. Iluf tet \&ant. . : . . . . . . . . . . . . . . . . . . 38

137. $35 r$ gRithe unb fein Rilfe. . . . . . . . . . . . . . . . . . . 33

138. Grabidrift ber Toditer elues freunbes, bie vor ber Faufe ftarb . . . . . 38

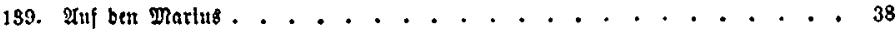

110. Iuf ben einäugigen Spicler Pfiff . . . . . . . . . . . . . . 39

141. In einen 2 tutor . . . . . . . . . . . . . . . . . . . 39

142. Tuf ben get . . . . . . . . . . . . . . . . . 99 


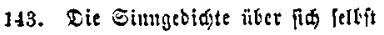

14. Xejuicb an ben sejer

Aำ)

Tarum t@ micter Epigramme madje. . . . . . . . . . . . . . . . . . . . 40

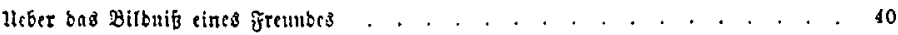

Jn ciu Stantmbud) . . . . . . . . . . . . . . . . . . . . . . . . . 40

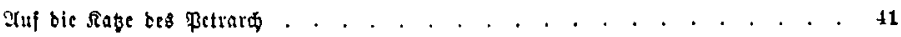

Grakfdrift auf Boltatren . . . . . . . . . . . . . . . . . . . . 41

Die 刃erlcumbung . . . . . . . . . . . . . . . . . . . . . . . 41

Jll eir Elammbud . . . . . . . . . . . . . . . . . . . . . . 42

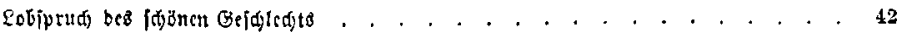

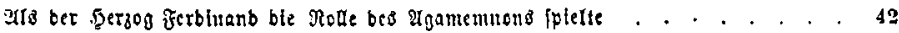

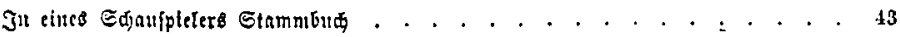

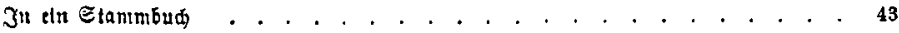

Sittenfurïde . . . . . . . . . . . . . . . . . . . . . . 43

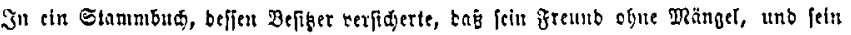

Mäbden elt Engel fry . . . . . . . . . . . . . . . . . . . . 41

\section{Qieber.}

Yin bie lezer. . . . . . . . . . . . . . . . . . . . . . . 47

Die Ramer . . . . . . . . . . . . . . . . . . . . . . . . . . 43

Dle א̃̈̈lfe .". . . . . . . . . . . . . . . . . . . . . 48

Die Betwifget . . . . . . . . . . . . . . . . . . . . . . . . 49

Die Betrübrif. Det Didgter nub fein f̈rcunb . . . . . . . . . . . . . . 49

भintwort eines trutluen Diळters . . . . . . . . . . . . . . . . . . 30

Das aufgebobente gebot . . . . . . . . . . . . . . . . . . . . . 50

Die Betcbliamteit . . . . . . . . . . . . . . . . . . . . 51

Dle Soustgaltung , . . . . . . . . . . . . . . . . . . . . . 51

Der Regen . . . . . . . . . . . . . . . . . . . . . . . . . 52

Die Gtärte bes $\mathfrak{B c i n b}$. . . . . . . . . . . . . . . . . . . . . 52

Der Sonberling . . . . . . . . . . . . . . . . . . . . . . . . 52

Der alte unb ber lunge \$itit . . . . . . . . . . . . . . . . . . . 53

Die Gürten . . . . . . . . . . . . . . . . . . . . . . . . . 58

2teranber . . . . . . . . . . . . . . . . . . . . 53

Dte Sdönc ron binten. . . . . . . . . . . . . . . . . . . . . . 54 
Yu ciut treine Edöne . . . . . . . . . . . . . . . . . . . . . . . . . 51

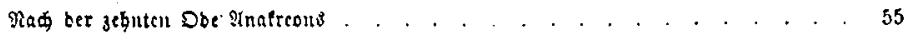

Das \$arabies . . . . . . . . . . . . . . . . . . . . 56

Die Jefpenfter . . . . . . . . . . . . . . . . . . . . . . . 56

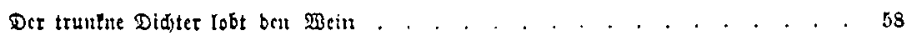

Sob det fraulgeit . . . . . . . . . . . . . . . . . . . . . . . 53

Die fauthcit . . . . . . . . . . . . . . . . . . . . 5s

Die \$lanetenberougher . . . . . . . . . . . . . . . . . . . . 59

Der Gefintace ber gltels . . . . . . . . . . . . . . . . . . . 59

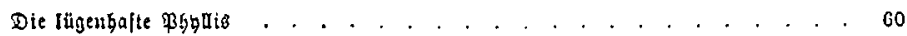

Die ficberumboiergigfte Doe stuntrecus . . . . . . . . . . . . . . . . 60

Radabmung biefer obe . . . . . . . . . . . . . . . . . . . . . 61

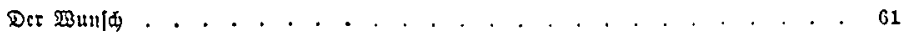

Der gröfte Mann . . . . . . . . . . . . . . . . . . 61

Der Jrt\$um . . . . . . . . . . . . . . . . . . . . . . 62

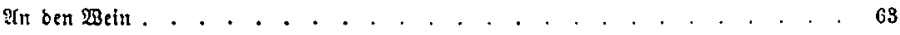

Phyats an Damion. . . . . . . . . . . . . . . . . . . 63

fothe wen id finge . . . . . . . . . . . . . . . . . . . . . . . 64

Die folafenbe snuta . . . . . . . . . . . . . . . . 65

Der Donner . . . . . . . . . . . . . . . . . . 66

Der mübige Pobel . . . . . . . . . . . . . . . . . . . . . . . 66

Die Mufit . . . . . . . . . . . . . . . . . . . . . . . 67

2tr bert 5oraz . . . . . . . . . . . . . . . . . . . . . . . G 6

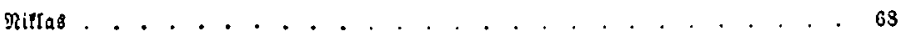

Die 凡üffe . . . . . . . . . . . . . . . . . . . 68

Der fóndrenbe \&lebgaber . . . . . . . . . . . . . . . . . 69

Trintlicb . . . . . . . . . . . . . . . . . . . . 69

Der \$ethuft . . . . . . . . . . . . . . . . . . . . 69

Det Benus . . . . . . . . . . . . . . . . . . . . . . . 60

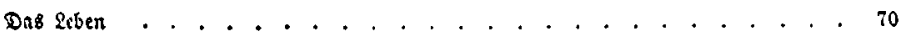

Dit Biene . . . . . . . . . . . . . . . . . . . . 71

Die liebe . . . . . . . . . . . . . . . . . . 71

Der KOD . . . . . . . . . . . . . . . . . . . . 72

Der fraule . . . . . . . . . . . . . . . . . . . 73

Der fitor . . . . . . . . . . . . . . . . . . . . . . 73

Dle wiber bert Cäar berfimorme Jgelben . . . . . . . . . . . . . . it

Die Ente . . . . . . . . . . . . . . . . . . . . . 75

Die bret Reide ber Ratur . . . . . . . . . . . . . . . . 76

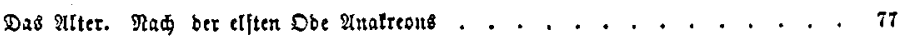

2tn bie Squalbe. Die jtoōtfte Obe Inalteonts . . . . . . . . . . . 77 
Eaite

Die Sunftridter unb bet Didter . . . . . . . . . . . . . . . . . is

QTu bie Sutuftridter . . . . . . . . . . . . . . . . . . . . T3

Anluatis.

Gieb aus bem Epanifden . . . . . . . . . . . . . . . . . . . . 79

Die Dicbin . . . . . . . . . . . . . . . . . . . . . 79

qublis. . . . . . . . . . . . . . . . . . . . . 19

Bacdus unb Đelena . . . . . . . . . . . . . . . . . . . . . . so

Pfn 2 thut . . . . . . . . . . . . . . . . . . . . . . 80

5etbenticb ber Epartanct . . . . . . . . . . . . . . . . . . . . 81

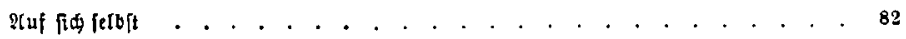

D de ll.

I. Der Gintritt bes 1752ften Jabreß . . . . . . . . . . . . 85

II. Ituf cine vornebme Bermäblung . . . . . . . . . . . . . . . 86

IIr. 2 Uúdicb cines frentibes . . . . . . . . . . . . . . . . . . . . 89

IV. $\mathfrak{A n}$ ben $\$$ gertm $\mathfrak{R}^{* *}$. . . . . . . . . . . . . . . . . . . . . 90

v. Der Tod eimes freunbes . . . . . . . . . . . . . . . . . . 91

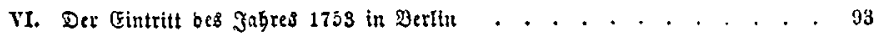

VII. Der 24fte Jenuer in Betlin . . . . . . . . . . . . . . . . . 94

vul. IIn feinen \$ruber . . . . . . . . . . . . . . . . . . . . 95

IX. Der Elittritt beß Jabres $175 \pm$ in Berlin . . . . . . . . . . . . 96

X. Der Eintritt bes Sabres 1755 in Berlin . . . . . . . . . . . . 97

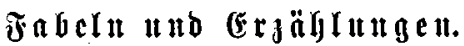

1. Der Sperting unb bie fielomars . . . . . . . . . . . . . 101

II. Der Mbler unb blc EuTe . . . . . . . . . . . . . . . . 101

UI. Der זanjbär . . . . . . . . . . . . . . . . . . . . . . 102

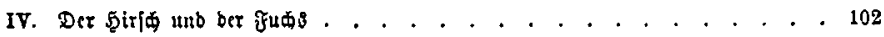

v. Die Sonte . . . . . . . . . . . . . . . . . . . . . . . 109

v1. Das Mufter ber Eben . . . . . . . . . . . . . . . . . . . 104

vII. Das Bebeimnis . . . . . . . . . . . . . . . . . . . . . 105

vIII. f̧auftin . . . . . . . . . . . . . . . . . . . 107

IX. Dte therthe Riebe . . . . . . . . . . . . . . . . . 107

X. Dte Bären . . . . . . . . . . . . . . . . . . . 108

XI. Der göroe unb bite grade . . . . . . . . . . . . . . . . 109

XIr. Dos fincifir . . . . . . . . . . . . . . . . . . . 110

XIII. Der Erentit . . . . . . . . . . . . . . . . . 111 
XIV. Eic Brifle . . . . . . . . . . . . . . . . . . . . . 120

Xv. Mix Bobenfitrom . . . . . . . . . . . . . . . . 123

\section{Fabelu. Drci $\mathfrak{B}$ ï djer.}

(Erfes $B \| d)$.

1. Die Erfdelnumg. . . . . . . . . . . . . . . . . . . 131

2. Der \$amfter utb ble Stmeife . . . . . . . . . . . . . . . . . . 132

3. Det gone unb ber saje . . . . . . . . . . . . . . . . . . . 132

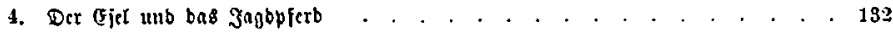

5. 3eus unb bas pletb . . . . . . . . . . . . . . . . . . . . 133

6. Der iffe unb ber Fuđs . . . . . . . . . . . . . . . . . . . . 134

7. Dle Raditigall unb ber $\mathfrak{P f a}$. . . . . . . . . . . . . . . . . 134

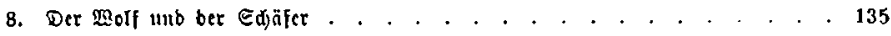

9. Das $\Re_{0 \beta}$ unb ber Gticr . . . . . . . . . . . . . . . . . . 135

10. Die Brille unb bie शadtigall . . . . . . . . . . . . . . . . . 195

11. Die Radjtigall unb ber 5gabldt. . . . . . . . . . . . . . . . . 136

12. Der etregetilde Molf . . . . . . . . . . . . . . . . . . . . 136

13. Der Pyönir . . . . . . . . . . . . . . . . . . 196

14. Dte Gans. . . . . . . . . . . . . . . . . . . . 137

15. Die Cide tnb has ङdjwein . . . . . . . . . . . . . . . . . . 137

16. Dic Bebpen . . . . . . . . . . . . . . . . . . . . . . . 198

17. Die Eperinge . . . . . . . . . . . . . . . . . . . . 138

18. Der Straús . . . . . . . . . . . . . . . . . . . . 198

19. Der Eperting unb ber Straub . . . . . . . . . . . . . . . . . 139

20. Die 5utnbe . . . . . . . . . . . . . . . . . . 189

21. Der. Judjs unb ber Gtord . . . . . . . . . . . . . . . . . , 140

22. Die Eute unb ber Edaghgrälier. . . . . . . . . . . . . . . . . 140

23. Die junge Sditarte . . . . . . . . . . . . . . . . . . . . 140

24. Merops . . . . . . . . . . . . . . . . . . . . . . 141

25. Der Bclitan . . . . . . . . . . . . . . . . . . . . 141

26. Ter Qüre unb ber Liger . . . . . . . . . . . . . . . . . . . 142

27. Det Eficr unt ber 5 fitja . . . . . . . . . . . . . . . . . . 142

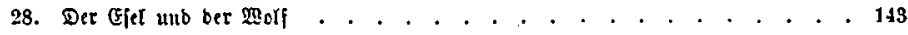

29. Det Epringer $\mathrm{im}$ Sdnd . . . . . . . . . . . . . . . . . . . 143

30. Tlefopus anb bec Fiel . . . . . . . . . . . . . . . . . 143

Zweites $B$ ud.

1. Die cberne Ditofüule . . . . . . . . . . . . . . . . . 144

2. 5erculc ... . . . . . . . . . . . . . . . 144 
3. Der funabe unb ble Edlange . . . . . . . . . . . . . . . . . 144

4. Der Miglf alf bem fodtuctte . . . . . . . . . . . . . . . . . 145

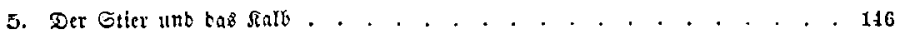

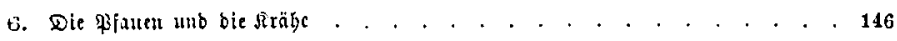

.. Det scme mit bom Ejal . . . . . . . . . . . . . . . . . . . 147

3. Der Efer mit ben \&ëtuen . . . . . . . . . . . . . . . . . . . 147

9. Die blinte 5ente . . . . . . . . . . . . . . . . . . . . . 11 t

10. Dic Eict . . . . . . . . . . . . . . . . . . . . . . . . 118

11. Das befdü̈zte \&amm . . . . . . . . . . . . . . . . . . . . . 119

12. Jupiter unb 2fyollo

13. Die SBafferfdilange . . . . . . . . . . . . . . . . . . . . . 149

14. Der fulde uttb bic karve . . . . . . . . . . . . . . . . . . . . 150

15. Der SRabe unt ber funt. . . . . . . . . . . . . . . . . . . 150

16. Der Geizige . . . . . . . . . . . . . . . . . . . . . . . 151

17. Der Pabe . . . . . . . . . . . . . . . . . . . . . . . 151

1s. Bens unb bas $e_{d j a f}$. . . . . . . . . . . . . . . . . . . . 152

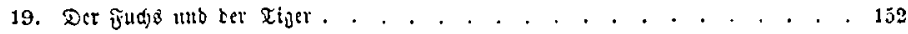

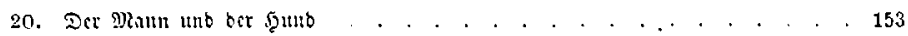

21. Dic זrnulic . . . . . . . . . . . . . . . . . . . . 153

2. Det fud . . . . . . . . . . . . . . . . . . . . . . . 154

23. D.s Sdaf . . . . . . . . . . . . . . . . . . . . . 154

24. Die 3 iegen . . . . . . . . . . . . . . . . . . . . . . . 155

25. Der witbe effeftranm . . . . . . . . . . . . . . . . . . 155

26. Det Şitfd unb ber fitchs . . . . . . . . . . . . . . . . . . 155

27. Det Dernftraud. . . . . . . . . . . . . . . . . . . . 156

28. Die futien . . . . . . . . . . . . . . . . . . . . . 156

29. হircliaz. . . . . . . . . . . . . . . . . . . . . . 157

30, Diinerba . . . . . . . . . . . . . . . . . 157

\section{Drittes Bur.}

1. Der Befiber bes Bogens . . . . . . . . . . . . . . . . . . . 158

2. Die Ntadtigall anb tic Rerdje . . . . . . . . . . . . . . . . 158

3. Dir Ocifít bes Salomo . . . . . . . . . . . . . . . . . . . 158

4. Dą Bejdut ber fochent . . . . . . . . . . . . . . . . . . . 159

5. Dns ๔djaf unb bie 巨dinalbe . . . . . . . . . . . . . . . . . 160

6. Der 9late. . . . . . . . . . . . . . . . . . 160

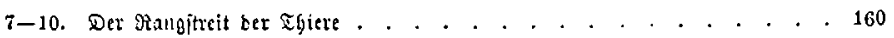

11. Der sär unb ocr Elephant . . . . . . . . . . . . . . . . . . 162

12. Фu Єtratis . . . . . . . . . . . . . . . . . . . . . . . 162 
Selte

13. 14. Die 230 hithaten . . . . . . . . . . . . . . . . . 163

15. Die Elitje . . . . . . . . . . . . . . . . . . . 163 16-22, Dic Gefdidte bes alten Wolfs . . . . . . . . . . . . . 164

23. Die Malls. . . . . . . . . . . . . . . . . . . . . . . . 168

24. Die Gdiwalbe . . . . . . . . . . . . . . . . . . . 168

25. Det Rlblet . . . . . . . . . . . . . . . . . . . . 168

26. Der funge unb ber alte Strid . . . . . . . . . . . . . . . . . 169

27. Der Pfau unt ker Salgn . . . . . . . . . . . . . . . . . . 169

28. Der 5̧itfd . . . . . . . . . . . . . . . . . . . . . 1G9

20. Der Abler unb ber fruds . . . . . . . . . . . . . . . . . 170

30. Der Gdjäfer unb ble naфtigall . . . . . . . . . . . . . . . 170

Mli Gara Gampion. Ein Traucrific . . . . . . . . . . . . . . 171 